\title{
Front Matter: Volume 11595
}

, "Front Matter: Volume 11595," Proc. SPIE 11595, Medical Imaging 2021: Physics of Medical Imaging, 1159501 (29 March 2021); doi:

$10.1117 / 12.2595450$

SPIE. Event: SPIE Medical Imaging, 2021, Online Only 


\title{
PROGRESS IN BIOMEDICAL OPTICS AND IMAGING
}

\section{Medical Imaging 2021 \\ Physics of Medical Imaging}

\author{
Hilde Bosmans \\ Wei Zhao \\ Lifeng Yu \\ Editors
}

\section{5-19 February 2021 \\ Online Only, United States}

Sponsored by

SPIE

Cooperating Organizations

AAPM-American Association of Physicists in Medicine (United States)

MIPS-Medical Image Perception Society (United States)

SIIM-Society for Imaging Informatics in Medicine (United States)

WMIS - World Molecular Imaging Society (United States)

Published by

SPIE

\section{Volume 11595}

Part One of Two Parts 
The papers in this volume were part of the technical conference cited on the cover and title page. Papers were selected and subject to review by the editors and conference program committee. Some conference presentations may not be available for publication. Additional papers and presentation recordings may be available online in the SPIE Digital Library at SPIEDigitalLibrary.org.

The papers reflect the work and thoughts of the authors and are published herein as submitted. The publisher is not responsible for the validity of the information or for any outcomes resulting from reliance thereon.

Please use the following format to cite material from these proceedings:

Author(s), "Title of Paper," in Medical Imaging 2021: Physics of Medical Imaging, edited by Hilde Bosmans, Wei Zhao, Lifeng Yu, Proceedings of SPIE Vol. 11595 (SPIE, Bellingham, WA, 2021) Sevendigit Article CID Number.

ISSN: 1605-7422

ISSN: 2410-9045 (electronic)

ISBN: 9781510640191

ISBN: 9781510640207 (electronic)

Published by

SPIE

P.O. Box 10, Bellingham, Washington 98227-0010 USA

Telephone +1 3606763290 (Pacific Time) · Fax +1 3606471445

SPIE.org

Copyright (c) 2021, Society of Photo-Optical Instrumentation Engineers.

Copying of material in this book for internal or personal use, or for the internal or personal use of specific clients, beyond the fair use provisions granted by the U.S. Copyright Law is authorized by SPIE subject to payment of copying fees. The Transactional Reporting Service base fee for this volume is $\$ 21.00$ per article (or portion thereof), which should be paid directly to the Copyright Clearance Center (CCC), 222 Rosewood Drive, Danvers, MA 01923. Payment may also be made electronically through CCC Online at copyright.com. Other copying for republication, resale, advertising or promotion, or any form of systematic or multiple reproduction of any material in this book is prohibited except with permission in writing from the publisher. The CCC fee code is $1605-$ $7422 / 21 / \$ 21.00$.

Printed in the United States of America by Curran Associates, Inc., under license from SPIE.

Publication of record for individual papers is online in the SPIE Digital Library.

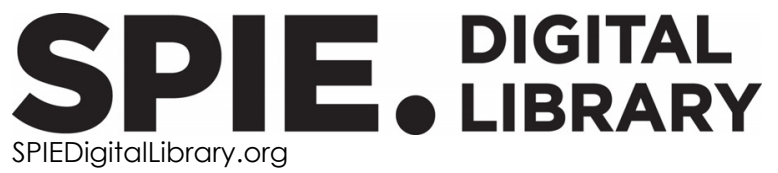

Paper Numbering: Proceedings of SPIE follow an e-First publication model. A unique citation identifier (CID) number is assigned to each article at the time of publication. Utilization of CIDs allows articles to be fully citable as soon as they are published online, and connects the same identifier to all online and print versions of the publication. SPIE uses a seven-digit CID article numbering system structured as follows:

- The first five digits correspond to the SPIE volume number.

- The last two digits indicate publication order within the volume using a Base 36 numbering system employing both numerals and letters. These two-number sets start with $00,01,02,03,04$, 05, 06, 07, 08, 09, 0A, OB ... 0Z, followed by 10-1Z, 20-2Z, etc. The CID Number appears on each page of the manuscript. 


\section{Contents}

\section{Part One}

\section{CT: OPTIMIZATION AND IMAGE QUALITY}

1159504 Multi-factorial optimization of imaging parameters for quantifying coronary stenosis in cardiac CT [11595-1]

1159505 A method for optimizing the x-ray fube current in ROI imaging using a simulation framework for radiation dose and image quality calculation for arbitrary fluence distributions [11595-2]

1159506 A framework to simulate CT images with tube current modulation [11595-3]

1159507 A web-based software platform for efficient and quantitative $\mathrm{CT}$ image quality assessment and protocol optimization [1 1595-4]

1159508 A Bayesian approach for CT perfusion parameters estimation with imperfect measurement [11595-5]

1159509 End-to-end modeling for predicting and estimating radiomics: application to gray level cooccurrence matrices in CT [11595-13]

PHOTON COUNTING: DETECTORS AND SYSTEMS

$11595 \mathrm{OB}$ Accurate characterization of metal implants and human materials using novel proton counting detector for Monte Carlo dose calculation in proton therapy [11595-7]

11595 OC Compton coincidence in silicon photon-counting CT detectors [11595-8]

11595 OD High resolution, full field-of-view, whole body photon-counting detector CT: system assessment and initial experience [11595-9]

11595 OE Characterization of a GaAs photon counting detector for mammography [11595-10]

\section{MACHINE LEARNING IN IMAGING PHYSICS}

$115950 G \quad$ A novel physics-based data augmentation approach for improved robust deep learning in medical imaging: lung nodule CAD false positive reduction in low-dose $C T$ environments [11595-14]

$11595 \mathrm{OH}$ Deep neural networks-based denoising models for CT imaging and their efficacy [11595-16] 
11595 Ol Training a low-dose CT denoising network with only low-dose CT dataset: comparison of DDLN and Noise2Void [1 1595-17]

$115950 \mathrm{~J} \quad$ A constrained Bregman framework for unsupervised convolutional denoising of multi-channel x-ray CT data [11595-18]

11595 OK A CT denoising neural network with image properties parameterization and control [11595-19]

$11595 \mathrm{OL} \quad$ Scatter distribution estimated and corrected by using convolutional neural network for multislice CT system [11595-20]

11595 OM Estimating Compton scatter distributions with a regressional neural network for use in a realtime staff dose management system for fluoroscopic procedures [11595-21]

\section{PHANTOMS AND LESION INSERTION}

11595 ON COPD quantifications via CT imaging: ascertaining the effects of acquisition protocol using virtual imaging trial [1 1595-15]

$1159500 \quad$ Generative adversarial networks and radiomics supervision for lung lesion synthesis (Robert F. Wagner All-Conference Best Student Paper Award) [11595-22]

11595 OP The creation of a breast cancer voxel model database for virtual clinical trials in digital breast tomosynthesis [11595-23]

$115950 Q \quad$ Computer model of mechanical imaging acquisition for virtual clinical trials [11595-24]

11595 OR 3D printed cardiac phantom for cardiac CT imaging performance evaluation and examination strategy verification [11595-25]

11595 OS Deep-learning lesion and noise insertion for virtual clinical trial in chest CT [1 1595-26]

IMAGE GUIDED INTERVENTION

11595 OT Evaluation of methods to derive blood flow velocity from $1000 \mathrm{fps}$ high-speed angiographic sequences (HSA) using optical flow (OF) and computational fluid dynamics (CFD) [1 1595-27]

11595 OU A hybrid photon counting and flat panel detector system for periprocedural hemorrhage monitoring in the angio suite [11595-28]

11595 OV A novel approach to calculating dual energy in the angiography suite [1 1595-29]

11595 OW Deformable image-based motion compensation for interventional cone-beam CT with a learned autofocus metric [11595-30]

11595 OX Cone-beam CT for neurosurgical guidance: high-fidelity artifacts correction for soft-tissue contrast resolution [11595-31] 
$115950 Z$ Positron emission tomography attenuation correction with dual energy CT electron density maps in the presence of iodinated contrast media [11595-33]

1159510 Back-end readout electronic design and initial results: a head-and-neck dedicated PET system based on CZT [1 1595-34]

$1159511 \quad$ Non-contact respiratory triggering for clinical MRI using frequency modulated continuous wave radar [11595-35]

1159512 A physics and learning-based transmission-less attenuation compensation method for SPECT [11595-36]

1159513 Wavelet improved GAN for MRI reconstruction [1 1595-37]

1159514 Correcting motion artifacts in MRI scans using a deep neural network with automatic motion timing detection [11595-38]

1159515 Monte Carlo simulation of an amorphous selenium-based multi-layer photon-counting detector for SPECT applications [1 1595-39]

\section{DETECTOR PHYSICS}

1159516 Improved optical quantum efficiency and temporal performance of a flat-panel imager with avalanche gain [11595-40]

1159517 Theoretical investigation of detector MTF of polycrystalline mercuric iodide x-ray converters incorporating Frisch grid structures for digital breast tomosynthesis [11595-41]

1159519 Spatial-frequency-dependent pulse height spectroscopy of x-ray scintillators using single x-ray imaging [1 1595-43]

115951 A A method to determine spectral performance of image post-processing algorithms [11595-44]

\section{SPECTRAL CT}

11595 1B Accurate physical density assessments from clinical spectral results [11595-45]

$115951 \mathrm{C}$ Quantitative dual-energy imaging in the presence of metal implants using locally constrained model-based decomposition [1 1595-46]

11595 1D Dual-contrast decomposition of dual-energy CT using convolutional neural networks [1 1595-47]

$11595 \mathrm{IE} \quad$ Generation of virtual non-contrast (VNC) image from dual energy CT scans using deep learning [11595-48] 
$11595 \mathrm{lF}$ Investigation of the linearized spectral contribution in the simultaneous estimation of spectra and basis images in multispectral CT reconstruction [1 1595-49]

$115951 G \quad$ Neural MLAA for PET-enabled dual-energy CT imaging [11595-50]

\section{BREAST TOMOSYNTHESIS}

$115951 \mathrm{H} \quad$ Strategies of improving lesion detection in wide-angle digital breast tomosynthesis (DBT) with angular dose distribution and detector design [11595-51]

1159511 Virtual clinical trial platforms for digital breast tomosynthesis: a local solution compared to the VICTRE plafform [11595-52]

$115951 \mathrm{~J}$ Development of magnification tomosynthesis for superior resolution in diagnostic mammography [11595-53]

$115951 \mathrm{~K} \quad$ Digital breast tomosynthesis denoising using deep convolutional neural network: effects of dose level of training target images [11595-54]

$11595 \mathrm{lL}$ Signal-to-noise ratio and contrast-to-noise ratio measurements for next generation tomosynthesis [11595-55]

\section{PHASE CONTRAST AND FLUORESCENCE IMAGING}

$115951 \mathrm{M}$ Incorporating dark-field information in mesh-based x-ray phase imaging [11595-56]

11595 iN Evaluation of edge-illumination and propagation-based $x$-ray phase contrast imaging methods for high resolution imaging application [1 1595-57]

\section{CT: RECONSTRUCTION}

$115951 Q \quad$ DeepInterior: new pathway to address the interior tomographic reconstruction problem in CT via direct backprojecting divergent beam projection data [1 1595-60]

11595 IR Manifold reconstruction of differences: a model-based iterative statistical estimation algorithm with a data-driven prior [11595-61]

11595 is Medical image reconstruction using compressible latent space invertible networks [11595-62]

$115951 \mathrm{~T} \quad$ IN-net: a spatiotemporal plus prior image-based convolutional neural network for 4D-CBCT reconstructions enhancement [11595-63]

$115951 \mathrm{U}$ Deep learning in image reconstruction: vulnerability under adversarial attacks and potential defense strategies [1 1595-64] 
$115951 \mathrm{X}$ Non-linear partial volume artifact reduction in spectral CT one step direct inversion reconstruction [1 1595-67]

$115951 \mathrm{Y}$ Stability of a spectral calibration model with non-linear intensity correction for photon-counting detectors [11595-68]

$1159512 \quad$ Analytical model for pulse pileup in photon counting detectors with seminonparalyzable behavior [1 1595-69]

1159520 Deep learning based spectral distortion correction and decomposition for photon counting CT using calibration provided by an energy integrated detector [11595-70]

\section{X-RAY IMAGING: DOSIMETRY, SCATTER, AND MOTION}

$1159521 \quad$ iPhantom: an automated framework in generating personalized computational phantoms for organ-based radiation dosimetry [11595-71]

1159522 Comparison of skin dose calculated by the dose tracking system (DTS) with a beam angular correction factor and that calculated by Monte-Carlo [1 1595-72]

1159523 The effect of underlying bone on the beam angular correction in calculating the skin dose of the head in neuro-interventional imaging [11595-73]

$1159524 \quad$ Preliminary in-vivo imaging evaluation of patient-specific scatter-corrected digital chest tomosynthesis [11595-74]

1159525 Image-domain cardiac motion compensation in multidirectional digital chest tomosynthesis [11595-75]

\section{DUAL-ENERGY: OPTIMIZATION AND CLINICAL APPLICATION}

1159526 Theoretical optimization of dual-energy x-ray imaging of chronic obstructive pulmonary disease (COPD) [11595-76]

1159527 Single-shot quantitative x-ray imaging from simultaneous scatter and dual energy measurements: a simulation study [11595-77]

1159528 Dual energy chest $x$-ray for improved COVID-19 detection using a dual-layer flat-panel detector: simulation and phantom studies [11595-78]

1159529 Assessment of reproducibility of volumetric breast density measurement using dual energy digital breast tomosynthesis [11595-79]

$115952 \mathrm{~A}$ Effects of $\mathrm{x}$-ray scatter in quantitative dual-energy imaging using dual-layer flat panel detectors [11595-80] 
$115952 \mathrm{~B} \quad$ Establishing a quality control protocol for dual-energy based contrast-enhanced digital mammography [11595-81]

POSTERS: ALGORITHM DEVELOPMENT

$115952 \mathrm{C}$ Classification of round lesions in dual-energy FFDM using a convolutional neural network: simulation study [11595-82]

$115952 \mathrm{D}$ Statistically adaptive filtering for low signal correction in $\mathbf{x}$-ray computed tomography [11595-83]

$115952 \mathrm{E}$ Accurate reconstruction of cross-section images from limited-angular-range data in humanlimb imaging [11595-84]

$115952 \mathrm{~F}$ Investigation of image reconstruction for digital breast tomosynthesis imaging by use of directional TV constraint [11595-85]

$115952 \mathrm{G}$ Improving presentation consistency of radiographic images using deep learning [11595-86]

$115952 \mathrm{H} \quad$ A feasibility study of data redundancy based on-line geometric calibration without dedicated phantom on Varian OBI CBCT system [11595-87]

$1159521 \quad$ Effects of smartphone sensor characteristics on dermatoscopic images: a simulation study [11595-88]

$115952 \mathrm{~J} \quad$ Quantifying the importance of spatial anatomical context in cadaveric, non-contrast enhanced organ segmentation [11595-89]

$115952 \mathrm{~K}$ A calibration method for conformal x-ray transmission imaging system [1 1595-90]

$115952 \mathrm{~L} \quad$ A hybrid domain approach to reduce streak artifacts of sparse view CT image via convolutional neural network [11595-91]

\section{Part Two}

\section{POSTERS: COMPUTED TOMOGRAPHY}

$115952 \mathrm{M}$ Optimization of $\mathrm{CT}$ angiography using physiologically-informed computational plaques, dynamic XCAT phantoms, and physics-based CT simulation [1 1595-100]

11595 2N Synthesizing high-resolution CT from low-resolution CT using self-learning [11595-101]

1159520 GAN-based sinogram completion for slow triple kVp switching CT [11595-102]

$115952 \mathrm{P} \quad$ Estimation of in vivo noise in clinical CT images: comparison and validation of three different methods against ensemble noise gold-standard [11595-103]

$115952 \mathrm{Q}$ Ultra-high-resolution CT and multi-resolution visualization of calcified coronary artery plaques: an ex vivo human study [11595-104] 
$115952 \mathrm{R} \quad$ Convolutional neural network based metal artifact reduction method in dental CT image [1 1595-105]

$115952 \mathrm{~S}$ Quantitative stability with patient size, dose, and kVp in spectral detector CT for pediatric imaging [1 1595-106]

$115952 \mathrm{~T} \quad$ Assessing the condition of spectral channelization (energy binning) in photon-counting CT: a singular value decomposition based approach [1 1595-107]

$115952 \mathrm{U} \quad$ Parameter-free Bayesian reconstruction for dual energy computed tomography [11595-108]

11595 2V Limited-angle CT reconstruction via data-driven deep neural network [11595-109]

11595 2W Rotating projection based localizer radiograph: attenuation calculation optimization and patient automatic centering with parallel-beam projection scheme [1 $1595-110]$

$115952 X \quad$ 3D VSHARP ${ }^{\circ}$, a general-purpose CBCT scatter correction tool that uses the linear Boltzmann transport equation [11595-111]

$115952 Y \quad$ Dose-length-product determination on cone beam computed tomography through experimental measurements and dose-area-product conversion [1 $1595-112]$

$115952 Z$ Sphere phantom approach to measure MTF of computed tomography system using convolutional autoencoder network [11595-113]

1159530 Automated patient-specific and organ-based image quality metrics on dual-energy CT datasets for large scale studies [1 1595-114]

$1159531 \quad$ Evaluation of a new low dose CВCT imaging protocol for measuring circumferential bone levels around dental implants [11595-115]

1159532 Stationary head CT scanner using CNT x-ray source arrays [11595-116]

1159533 The impact of CT-data segmentation variation on the morphology of osteological structure [11595-117]

1159534 Correlation of respiratory changes in lung density on dynamic chest radiographs with changes in the CT value: a computational phantom study [1 1595-118]

POSTERS: X-RAY IMAGING

1159535 Mobile x-ray tomography system with intelligent sensing for 3D chest imaging [11595-167]

1159536 Stationary head CT with linear CNT x-ray source arrays: image quality assessment through simulation [11595-168]

1159538 Investigation of the need for an x-ray scatter-reduction grid during neurointerventional procedures [11595-170] 
$1159539 \quad X$-ray tube based on carbon nanotube field emitter for low dose mini C-arm fluoroscopy [11595-171]

11595 3B Stent enhancement using marker response network and focus conversion learning in x-ray fluoroscopy sequences [11595-173]

$115953 \mathrm{C}$ Characterization of compact alumina vacuum sealed $x$-ray tube for medical imaging: interpretation with simulation program [11595-174]

11595 3D Theoretical comparison of the detective quantum efficiency of halide lead perovskite, cesium iodide and selenium $x$-ray imaging detectors [11595-175]

11595 3E Thickness uniformity and stability of amorphous selenium films on flexible substrates for indirect conversion x-ray detection [11595-176]

11595 3F Development of a novel algorithm to improve image quality in chest digital tomosynthesis using convolutional neural network with super-resolution [1 1595-177]

$115953 \mathrm{G}$ Stationary multi x-ray source system with carbon nanotube emitters for digital tomosynthesis [11595-178]

$115953 \mathrm{H} \quad$ Estimation of lung volume changes from frontal and lateral views of dynamic chest radiography using a convolutional neural network model: a computational phantom study [11595-179]

$1159531 \quad$ An automatic approach to lung region segmentation in chest $x$-ray images using adapted U-Net architecture [1 1595-180]

11595 3J Development of microfocus X-ray source based on CNT emitter for intraoperative specimen radiographic system [11595-181]

11595 3K An analysis of radiomics features in lung lesions in COVID-19 [11595-182]

$115953 \mathrm{~L} \quad$ A parametric fitting technique for rapid determination of a skin-dose correction factor for angle of beam incidence during image-guided endovascular procedures [1 1595-183]

POSTERS: IMAGE RECONSTRUCTION

$115953 \mathrm{M} \quad$ Novel reconstruction algorithms that facilitate real time 4D tomosynthesis [1 1595-119]

$115953 \mathrm{~N} \quad$ MLEM reconstruction with specific initial image for cone-beam x-ray luminescence computed tomography [11595-120]

1159530 Quantitative texture analysis of normal and abnormal lung tissue for low dose CT reconstruction using the tissue-specific texture prior [11595-121]

11595 3P Estimation of statistical weights for model-based iterative CT reconstruction [1 1595-122]

$115953 Q \quad$ Deep learning-based sinogram extension method for interior computed tomography [11595-123] 
11595 3R Quantitative cone-beam x-ray luminescence computed tomography with 3D TV denoising based on Split Bregman method [1 1595-124]

11595 3S 3D image reconstruction for symmetric-geometry CT with linearly distributed source and detector in a stationary configuration [1 1595-125]

11595 3T Deep-learning based joint estimation of dual-tracer PET image activity maps and clustering of time activity curves [1 1595-126]

$115953 \mathrm{U} \quad$ A new method and system for fast and accurate 3D projections in medical imaging and iterative reconstruction [11595-127]

11595 3V Model-based reconstruction algorithm for x-ray induced acoustic tomography [11595-128]

POSTERS: MACHINE LEARNING APPLIED TO IMAGING PHYSICS

11595 3W A deep learning approach to correctly identify the sequence of coincidences in cross-strip CZT detectors [11595-137]

$115953 \mathrm{X}$ Learning a projection operator onto the null space of a linear imaging operator [11595-138]

115953 A probabilistic conditional adversarial neural network to reduce imaging variation in radiography (Cum Laude Poster Award) [11595-139]

1159532 Self-supervised learning for CT deconvolution [11595-140]

1159540 Generation of contrast-enhanced CT with residual cycle-consistent generative adversarial network (Res-CycleGAN) [11595-141]

1159542 Investigation of the efficacy of a data-driven CT artifact correction scheme for sparse and truncated projection data for intracranial hemorrhage diagnosis [11595-143]

$1159543 \quad$ Estimation of patient eye-lens dose during neuro-interventional procedures using a dense neural network (DNN) [11595-144]

$1159544 \quad$ Low-dose CT denoising via CNN with an observer loss function [1 1595-145]

1159545 CNN-based CT denoising with an accurate image domain noise insertion technique [11595-146]

1159546 A deep learning post-processing to enhance the maximum likelihood estimate of three material decomposition in photon counting spectral CT [11595-147]

1159547 Synthetic dual energy CT imaging from single energy CT using deep attention neural network [11595-148]

1159548 Image reconstruction from projections of digital breast tomosynthesis using deep learning [11595-149] 
1159549 Transfer learning-based synthetic CT generation for MR-only proton therapy planning in children with pelvic sarcomas [1 1595-150]

11595 4A PET image resolution uniformity improvements using deep learning [11595-151]

11595 4B Enhanced PET/MRI reconstruction via dichromatic interpolation of domain-translated zerodose PET [1 1595-152]

11595 4C A topological approach for the pattern analysis on chest $x$-ray images of COVID-19 patients [11595-153]

11595 4D Deep learning based similarity-consistency abnormality detection (SCAD) model for classification of MRI patterns of multiple myeloma (MM) infiltration [1 1595-154]

$115954 \mathrm{E} \quad$ Using a convolutional neural network for human recognition in a staff dose management software for fluoroscopic interventional procedures [1 1595-155]

11595 4F Improving proton CT beyond iterative methods with a convolutional neural network [11595-156]

\section{POSTERS: PHOTON COUNTING AND PHASE CONTRAST IMAGING}

11595 4G Low-dose photon-counting CT with penalized-likelihood basis-image reconstruction: image quality evaluation [1 1595-157]

$115954 \mathrm{H} \quad$ Maximum A Posteriori material decomposition for spectral photon-counting CT: application to human blood iron level estimation [1 1595-158]

$1159541 \quad$ Multivariate SNR in spectral computed tomography [11595-159]

$115954 \mathrm{~J}$ Theoretical comparison of energy-resolved and temporal-subtraction angiography [11595-160]

$115954 \mathrm{~K} \quad$ An experimental evaluation of material separability in photon-counting CT [11595-161]

$115954 \mathrm{M}$ Towards $\mathrm{x}$-ray phase contrast tomography in clinical conditions: simulation and phase retrieval development [11595-163]

$115954 \mathrm{~N} \quad$ Tracking cells in the brain of small animals using synchrotron multi-spectral phase contrast imaging [1 1595-164]

1159540 Challenges of employing a high resolution x-ray detector in a coded-aperture x-ray phasecontrast imaging system [1 1595-165]

$115954 \mathrm{P} \quad$ Quantitative phase retrieval simulation for mesh-based $\mathbf{x}$-ray phase imaging systems [11595-166] 
$115954 Q \quad$ Assessment of a tumour growth model for virtual clinical trials of breast cancer screening [11595-92]

$115954 R$ Iterative method to achieve noise variance stabilization in single raw digital breast tomosynthesis [11595-93]

11595 4S Computational model of tumor growth for in silico trials [11595-94]

$115954 \mathrm{~T} \quad$ Accuracy and precision of a structured light scanning system for acquiring 3D information of the breast under compression [11595-95]

$115954 \mathrm{U}$ Clinical study for evaluation of an inflatable air cushion to reduce patient discomfort during tomosynthesis compression [11595-96]

$115954 \mathrm{~V} \quad$ Next generation tomosynthesis image acquisition optimization for dedicated PET-DBT attenuation corrections [1 1595-97]

$115954 \mathrm{~W}$ Analysis of digital breast tomosynthesis acquisition geometries in sampling Fourier space [11595-98]

11595 4X Realism of mammography tissue patches simulated using Perlin noise: a forced choice reading study [11595-99]

\section{POSTERS: IMAGING METHOD}

$115954 \mathrm{Y}$ Estimation of the dynamic volume of each lung via rapid limited-slice dynamic MRI [11595-129]

$115954 Z$ Internal layer illumination method and apparatus for medical imaging by multiple beam interference [11595-130]

1159550 Electrode displacement elastography for differentiating metastatic liver cancer microwave ablation procedures [11595-131]

$1159551 \quad$ Proton imaging with machine learning [11595-132]

1159552 Ring artifact and non-uniformity correction method for improving XACT imaging [11595-133]

1159553 Thermal interpretation procedure for the adjunct detection of thyroid pathologies [1 1595-134]

1159554 Realistic head modeling of electromagnetic brain activity: an integrated Brainstorm-DUNEuro pipeline from MRI data to the FEM solutions [11595-135]

1159555 Modified sensitivity, noise equivalent count rate performance, and scatter fraction measurements of asymmetrical and dedicated brain positron emission tomographs [11595-136] 
Proc. of SPIE Vol. 11595 1159501-14

Downloaded From: https://www.spiedigitallibrary.org/conference-proceedings-of-spie on 26 Apr 2023 Terms of Use: https://www.spiedigitallibrary.org/terms-of-use 